\title{
Divergent tail and throat ornamentation in the barn swallow across the Japanese islands
}

\author{
Masaru Hasegawa $\cdot$ Emi Arai
}

Received: 28 July 2012/ Accepted: 16 October 2012/Published online: 2 November 2012

(C) Japan Ethological Society and Springer Japan 2012

\begin{abstract}
The geographic variations in male ornamentation provide insights into how different populations reach a different mean trait value under opposing forces of natural and sexual selection. Although the latitudinal cline of the elongated tail streamer, a sexually selected trait in the European subspecies of the barn swallow Hirundo rustica rustica, is a classic example, it has recently been shown that other subspecies of swallows have different targets of sexual selection. Here, we studied the latitudinal cline of ornamentation in the Asian subspecies, H. r. gutturalis, in which not the tail length but the white tail spot and red throat patch are important sexually selected traits. After controlling for covariates, the size of the white tail spot increased with latitude, while the size of the red throat patch decreased with latitude. On the other hand, we could not find any clear pattern regarding the elongated tail streamer, measured as fork depth. The divergent ornamentation across populations could be explained by latitudinal clines of sexually selected advantages of each ornament.
\end{abstract}

Keywords Multiple ornaments - Red throat patch · Fork tail · White tail spot $\cdot$ Sexual selection

Electronic supplementary material The online version of this article (doi:10.1007/s10164-012-0352-y) contains supplementary material, which is available to authorized users.

M. Hasegawa $(\square)$

2-16-15 Nagata, Kanazawa, Ishikawa 920-0043, Japan

e-mail: perorobomusadiobe@gmail.com

E. Arai

Division of Ecology and Evolutionary Biology, Graduate School of Life Sciences, Tohoku University, Sendai 980-8578, Japan

\section{Introduction}

The geographic variations in male ornamentation provide insights into how different populations reach a different mean trait value under opposing forces of natural and sexual selection. A classic example can be observed in the European subspecies of the barn swallow, Hirundo rustica rustica (Møller 1994). Male swallows obtain sexually selected advantages from displaying elongated tail streamers at the cost of impaired foraging. The fact that males display elongated streamers in the more northern regions of Europe may be explained by the observation that foraging for flying insects is more expensive in southern than in northern Europe because of the greater flying ability of the insects with high ambient temperatures. Therefore, males exhibit elongated tail streamers in the north where natural selection may oppose sexual selection to a lesser extent (Møller 1994).

Recently, it has been shown that other subspecies of the barn swallow have different targets of sexual selection (Safran and McGraw 2004; Neuman et al. 2007; see also Vortman et al. 2011). In the Asian subspecies, H. r. gutturalis, which have short tail streamers (Turner 2006; Hasegawa et al. 2010), sexual selection for tail streamers is not strong (e.g., Kojima et al. 2009; Hasegawa et al. 2010). Instead, previous studies have shown that the white tail spot and red throat patch are the two important sexually selected traits (Hasegawa et al. 2010, 2012; reviewed in Hasegawa 2011). Thus, if there are any geographic variations in male ornamentation, these two male ornaments would have geographic variations in this subspecies rather than tail streamers. As in the European subspecies (Møller 1994), we studied the latitudinal cline of male ornamentation in the Japanese islands by using a dataset of skin specimens preserved in museums. We also discuss the potential reasons for the observed pattern. 


\section{Materials and methods}

\section{Study specimens}

We used skin specimens that were collected during the breeding season (i.e., from April to August; Hasegawa et al. 2010; reviewed in Turner 2006) to exclude migrating or wintering swallows. In total, we examined 65 skins of adult barn swallows from six museums. The recovery site, as written on the label of each specimen, was classified into three groups, South, Middle, and North, which corresponded to the latitude. South consists of the Kyushu region (traditionally called "Minami-nippon" meaning southern Japan; Tanioka 2007); North consists of Hokkaido Prefecture and Tohoku region (traditionally called "Kita-nippon" meaning northern Japan; Tanioka 2007); and Middle includes all prefectures between the North and South (see supplementary material S1). This three-group classification was chosen due to small sample size (i.e., samples were too scattered to analyze using original latitude), with reference to the climate, geography, as well as latitude. This classification was closely linked to the mean annual temperature, i.e., decreasing mean annual temperature toward the north ( $n=16$; Spearman's rank correlation coefficient, $r_{\mathrm{s}}=$ $-0.85, P<0.001$; see supplementary material S1; Japan Meteorological Agency 2011). This classification would be enough large to capture the general geographic trends of ornamentation as dispersal distances (and perhaps gene flow, too) of barn swallows rarely exceed $200 \mathrm{~km}$ (max. $410 \mathrm{~km}$, usually a few kilometers; reviewed in Turner 2006), which is much shorter than the distance between the two boundaries (i.e., boundary of North and Middle and that of Middle; ca. $800 \mathrm{~km}$ ). The sex of the swallows was determined on the basis of the morphology and information written on the label of each specimen.

\section{Measurements}

We measured the wing length, tail fork depth, and sizes of the white tail spot and the throat patch to the nearest millimeter. Because of the difficulty in measuring from the skin specimens, we used indices that were somewhat different from those used for live birds (see Hasegawa et al. 2010). The tail fork depth was used instead of the tail length for the easiness and preciseness of measurements, as the tail fork depth of males also increases from south to north at least in Europe (Møller 1995; Møller et al. 1995; Barbosa and Møller 1999; reviewed in Turner 2006, p. 24). This procedure would not be problematic, as the tail fork depth was highly significantly correlated with our measure of tail length after controlling for sex and site ( $n=55$, Pearson's partial correlation coefficient, $\left.r_{\mathrm{p}}=0.91, P<0.0001\right)$. In fact, similar results were obtained when using our poor measure of tail length instead of the tail fork depth with and without controlling for wing length (data not shown). To avoid destroying the skin specimen, only the outermost white tail spot was measured. Rather than the absolute height, the relative size of the red throat patch [i.e., the height of the red throat patch/(height of the red throat patch and black breast band)] was measured to control for different specimen postures (imagine shrunken and stretched neck specimens). This procedure is justified because of the following reasons. First, qualitatively similar results could be obtained when using the absolute height of red throat patch (data not shown). Second, the denominator (i.e., the height of the red throat patch and black breast band) was considerably constant (variance $=4.95$ ) compared to the absolute height of the red throat patch (variance $=48.77 ; F$ test, $F=2.85$, $P<0.01$; Joetsu population, $n=34$; M. Hasegawa, unpublished data). Finally, there was no geographic cline in the denominator with and without controlling for sex and/or other morphologies ( $n=46$, ICoef.I $<0.18, F<0.03, P>0.87$ ), indicating that controlling for the denominator would not confound the results. In this study, we did not analyze plumage coloration because of plumage color fading (e.g., Hasegawa et al. 2008), and hence, we regarded the throat patch size as a representative of the colorfulness of the red throat patch. This view is justified, because throat patch size and patch coloration are developmentally and functionally integrated, at least in part (e.g., Hill 2002; reviewed in Hill and McGraw 2006a, b; see also supplementary material S2).

\section{Statistics}

Body measurements were analyzed using a general linear model (GLIM). The significance of the terms in a GLIM was based on the difference in deviance and degrees of freedom of the models with and without the predictor in question. In the analysis, we fitted a full model containing all explanatory variables and covariates that were supposed to have an influence on body measurements (i.e., variables inter-correlated with the focused measurement). Statistical control of inter-correlation is justified, as life-history theory predicts that high-quality individuals can invest large on both two traits that were functionally correlated (i.e., sexually selected traits in the current case; see "Results"), which would obscure the large-scale pattern of focused traits (sensu Stearns 1992; Andersson et al. 2002). Collinearity between variables can cause problems in GLIM, but this is generally not considered problematic when $|r|<0.70$, as in the present study (cf. Møller 2004). A final model was selected by progressively eliminating non-significant explanatory variables $(P>0.05)$. In the analysis of inter-correlation among body measurements, we used Pearson's partial correlation coefficient to control for sex and site. All data analyses were performed using the $\mathrm{R}$ statistical package (v.2.8.0; R Development Core Team 
2008). Throughout the paper, statistical tests are two-tailed and $P$ values lower than 0.05 are considered as significant.

\section{Results}

Inter-correlations among measurements

After controlling for sex and site, a positive relationship was observed between the sizes of the white tail spot and throat patch ( $n=46$, Pearson's partial correlation coefficient, $\left.r_{\mathrm{p}}=0.39, P<0.01\right)$, indicating that swallows with large white tail spot also had large throat patch for each sex in each site. Similarly, a non-significant positive relationship was observed between the tail fork depth and wing length $\left(n=58, r_{\mathrm{p}}=0.25, P=0.06\right)$. The other relationships among wing length, sizes of the white tail spot and throat patch, and tail fork depth were far from significant $\left(n>43,\left|r_{\mathrm{p}}\right|<0.21, P>0.16\right)$.

Wing length

Wing length was significantly related to sex, but it was not significantly related to site (Table 1); across the Japanese islands, males showed longer wing lengths than females.

\section{Tail fork depth}

Tail fork depth was significantly related to sex, but it was not significantly related to site (Table 2). This was also the case even after including a marginally significant relationship with the wing length in the final model (data not shown). Overall, males had on average deeper forks than females.

The size of the white tail spot

After controlling for the inter-correlation with the throat patch size, the size of the white tail spot was significantly related to two variables; sex and site (Table 3 ). The size of the white tail spot was larger in males than in females, and it increased toward the north (Fig. 1). In the north, swallows had white tail spots that were, on an average, $3.0 \mathrm{~mm}$ larger than those in the south, with anything else being equal. This difference corresponds to ca. $14 \%$ of the total length of the white tail spot.

The size of the red throat patch

After controlling for inter-correlation with the size of the white tail spot, throat patch size was significantly related to site (Table 4). Throat patch size decreased toward the north (Fig. 2). In the north, swallows had throat patches that were, on an average, $17 \%$ smaller red throat in relation to
Table 1 GLIM explaining wing length of the Asian barn swallow, Hirundo rustica gutteralis $(n=64)$

\begin{tabular}{llrll}
\hline & $d f$ & Coef. & $F$ & $P$ \\
\hline Sex $^{\mathrm{a}}$ & 1 & -1.93 & 5.36 & 0.02 \\
Latitude & 1 & 0.75 & 1.68 & 0.20 \\
\hline
\end{tabular}

Mean wing length $\pm \mathrm{SD}=115 \pm 3 \mathrm{~mm}$. Coefficients, $F$ values, and $P$ values of significant terms $(P \leq 0.05)$ are from the final model, whereas coefficients, $F$ values, and $P$ values of non-significant terms are from the final model with each non-significant term added separately. The interaction term was excluded because of non-significance

${ }^{a}$ Indicates the variables retained in the final model

Table 2 GLIM explaining fork depth $(n=58)$

\begin{tabular}{llrrc}
\hline & $d f$ & \multicolumn{1}{c}{ Coef. } & \multicolumn{1}{c}{$F$} & \multicolumn{1}{c}{$P$} \\
\hline Sex $^{\text {a }}$ & 1 & -10.58 & 31.05 & $<0.0001$ \\
Latitude & 1 & 0.67 & 0.04 & 0.61 \\
Wing length & 1 & 0.53 & 3.89 & 0.054 \\
\hline
\end{tabular}

Mean fork depth $\pm \mathrm{SD}=42 \pm 9 \mathrm{~mm}$

See note to Table 1

Table 3 GLIM explaining the size of the white tail spot $(n=46)$

\begin{tabular}{llrrc}
\hline & $d f$ & Coef. & \multicolumn{1}{c}{$F$} & $P$ \\
\hline Sex $^{\mathrm{a}}$ & 1 & -5.28 & 33.60 & $<0.0001$ \\
Latitude $^{\mathrm{a}}$ & 1 & 1.76 & 5.04 & 0.03 \\
Throat patch size $^{\mathrm{a}}$ & 1 & 10.19 & 7.72 & $<0.01$ \\
\hline
\end{tabular}

Mean size of white tail spot $\pm \mathrm{SD}=21 \pm 4 \mathrm{~mm}$

See note to Table 1

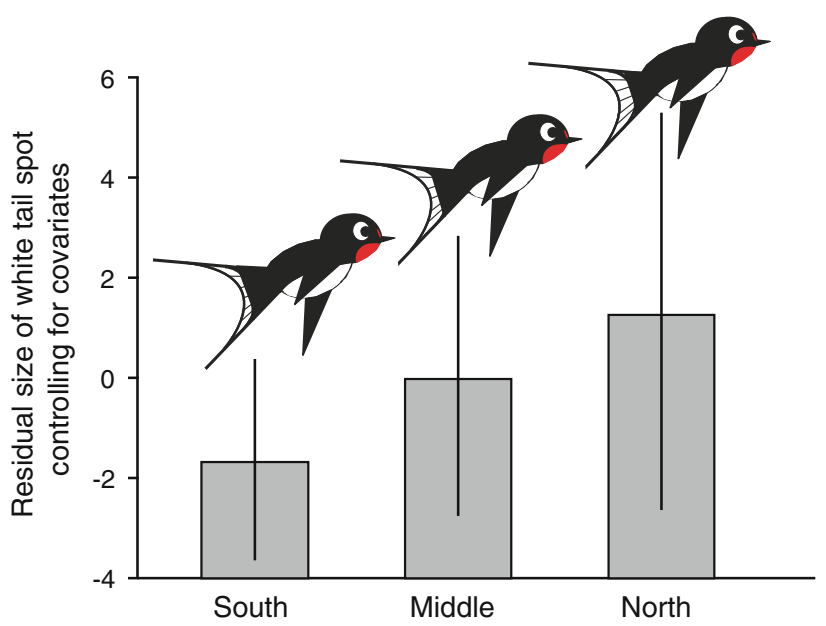

Fig. 1 Relationship between the site and residual size of the white tail spot of the Asian barn swallow, Hirundo rustica gutteralis after controlling for significant covariates (see Table 3). Bars mean \pm SD

total size of throat (i.e., height of the red throat patch and black breast band) than throat patches of swallows in the south, with anything else being equal. 
Table 4 GLIM explaining throat patch size $(n=46)$

\begin{tabular}{lrrrr}
\hline & $d f$ & Coef. & \multicolumn{1}{c}{$F$} & \multicolumn{1}{c}{$P$} \\
\hline Sex & 1 & 0.06 & 1.54 & 0.22 \\
Latitude $^{\mathrm{a}}$ & 1 & -0.09 & 10.56 & $<0.01$ \\
The size of white tail spot $^{\mathrm{a}}$ & 1 & 0.02 & 6.70 & 0.01 \\
\hline
\end{tabular}

Mean throat patch size $\pm \mathrm{SD}=0.85 \pm 0.13$, measured as the height of the red throat patch/(height of the red throat patch and black breast band); see "Materials and methods"

See note to Table 1

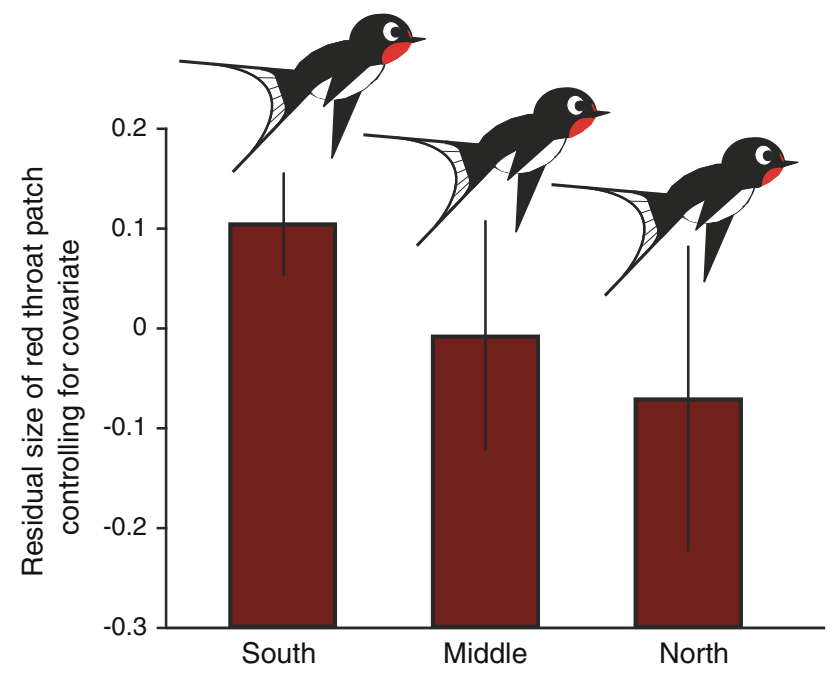

Fig. 2 Relationship between the site and residual size of the red throat patch after controlling for a significant covariate (see Table 4). Bars mean $\pm \mathrm{SD}$

\section{Discussion}

These findings indicate latitudinal clines of two ornaments, throat patch size and white tail spot size, after controlling for inter-correlation. Since the barn swallow is a migratory bird species, restricted gene flow among populations would not be an appropriate explanation for the observed pattern (see also Møller et al. 2006). Rather, the observed pattern can be explained by the relative importance of the two ornaments.

A potential explanation for the geographic variation in ornamentation is that natural selection favors different ornaments with latitude. Because neither ornament impairs aerodynamic ability, the explanation of foraging costs (sensu Møller 1994) could not be applied. Likewise, the explanation of thermoregulation would be unlikely because both ornaments consist of small patches and are positioned at hidden places (i.e., throat patch: ventral side; white tail spot: between tails; Turner 2006; see also Figs. 1, 2). Another explanation is the physiological costs of the ornaments, as predicted by sexual selection theory
(Andersson 1994). In fact, white tail spots have a cost of reduced resistance to ectoparasites (Kose and Møller 1999; Kose et al. 1999), and the red throat patch has a hormoneregulated cost (Safran et al. 2008; Eikenaar et al. 2011). Unfortunately, we do not have a clear prediction of the geographic variation in these costs, which needs to be studied in the future.

An alternative explanation for the geographic variation in ornamentation is that sexual selection favors different ornaments with latitude. Although previous studies showed that the two ornaments are sexually selected, they were related to each sexual selection episode in different manners. For example, a large white tail spot brings early breeding onset to the owner (Hasegawa et al. 2010; Hasegawa 2011), which would be a greater help in northern populations, where the breeding period is restricted (Turner 2006). In addition, males with a large white tail spot have the reproductive advantage at incubation periods in high latitudes (i.e., cold environments) because of the effective incubation investment of their mates (Hasegawa et al. 2012). In contrast, the red throat patch is only weakly related to breeding onset date (Hasegawa et al. 2010; Hasegawa 2011), indicating that, at high latitudes, colorful throat patches are not so beneficial to owners as large white tail spots. Rather, males with a colorful throat had a reproductive cost at high latitudes because of the ineffective incubation investment of their mates: females paired to these colorful males had longer off-bout durations, which elongates the incubation period, compared with other females (Hasegawa et al., in preparation; see also Hasegawa et al. 2012). Instead, males with a colorful throat patch had a higher probability of having multiple clutches (supplementary material S2) and a high-quality territory (Hasegawa 2011), perhaps because of high aggressiveness (Safran et al. 2008; Eikenaar et al. 2011). Colorful throat patches would be relatively beneficial at lower latitudes where these components rather than breeding onset date would be more important than at higher latitudes (Turner 2006).

These arguments assume situations with no tradeoff between the two ornaments, but this might not be the case. Individuals may often be forced to trade off the resources they allocate to different ornaments which would be costly to produce and maintain (e.g., Andersson et al. 2002; Wagner et al. 2012). With such a tradeoff between two ornaments, the geographic cline of one ornament will automatically cause a geographic cline of the other ornament in the reverse direction. The positive correlation between throat patch size and the size of the white tail spot within sites at first seems to be inconsistent with the tradeoff explanation. However, within-site patterns do not always predict the tradeoff among ornaments, as highquality individuals can invest significantly in both two 
traits that are functionally correlated, which would mask the pattern of resource allocation (sensu Stearns 1992; Andersson et al. 2002). The validation of the tradeoff explanation requires experiments (e.g., Wagner et al. 2012), which are clearly beyond our scope here. It should be noted that this tradeoff explanation and the above explanations are not mutually exclusive and can work together.

Here, we showed the latitudinal cline of the two sexually selected traits, red throat patch and white tail spot, across the Japanese islands. Because the range of this study was smaller than that of the European study (supplementary material S1; see also Møller 1994, 1995), we could not directly compare the intensity of the latitudinal cline of tail fork depth in the two subspecies. Rather, it should be noted that we found divergent ornamentation even in the small study range. The latitudinal cline of the sexually selected advantages of each ornament could explain the divergent ornamentation, which would be maintained without speciation unless the targets of sexual selection change.

Acknowledgments We are grateful to The Museum of Nature and Human Activities, Hyogo, Miyazaki Prefectural Museum of Nature and History, Yamashina Institute for Ornithology, National Museum of Nature and Science, Tokyo, Kitakyushu Museum of Natural History \& Human History, Hokkaido University Natural History Museum. We also thank Isao Nishiumi, Kiyoaki Ozaki, Sayaka Kobayashi, Naoko Endo, Toyofumi Sueyoshi, Sunao Suzuki, Yutaka Nakamura, Zenji Takeishi, and Masaru Kato.

\section{References}

Andersson M (1994) Sexual selection. Princeton University Press, Princeton

Andersson S, Pryke SR, Ornborg J, Lawes MJ, Andersson M (2002) Multiple receivers, multiple ornaments, and a trade-off between agonistic and epigamic signaling in a widowbird. Am Nat 160:683-691

Barbosa A, Møller AP (1999) Sexual selection and tail streamers in the barn swallow: appropriate tests of the function of sizedimorphic long tails. Behav Ecol 10:112-114

Eikenaar C, Whitham M, Komdeur J, van der Velde M, Moore IT (2011) Testosterone, plumage colouration and extra-pair paternity in male North-American barn swallows. PlosOne 6:e23288. doi:10.1371/journal.pone. 0023288

Hasegawa M (2011) Sexual selection on multiple ornaments in barn swallow. PhD thesis, University of Tsukuba

Hasegawa M, Arai E, Watanabe M, Nakamura M (2008) Methods for correcting plumage color fading in the Barn Swallow. Ornithol Sci 7:117-122

Hasegawa M, Arai E, Watanabe M, Nakamura M (2010) Mating advantage of multiple male ornaments in the Barn Swallow Hirundo rustica gutturalis. Ornithol Sci 9:141-148
Hasegawa M, Arai E, Watanabe M, Nakamura M (2012) High incubation investment of females paired to attractive males in barn swallows. Ornithol Sci 11:1-8

Hill GE (2002) A red bird in a brown bag: the function and evolution of colorful plumage in the house finch. Oxford University Press, Oxford

Hill GE, McGraw KJ (2006a) Bird coloration. I. Mechanisms and measurements. Harvard University Press, Cambridge

Hill GE, McGraw KJ (2006b) Bird coloration. II. Function and evolution. Harvard University Press, Cambridge

Japan Meteorological Agency (2011) http://www.data.jma.go.jp/obd/ stats/data/stat/tenko2011.pdf

Kojima W, Kitamura W, Kitajima S, Ito Y, Ueda K, Fujita G, Higuchi H (2009) Female barn swallows gain indirect but not direct benefits through social mate choice. Ethology 115:939-947

Kose M, Møller AP (1999) Sexual selection, feather breakage and parasites: the importance of white spots in the tail of the barn swallow. Behav Ecol Sociobiol 45:430-436

Kose M, Mand R, Møller AP (1999) Sexual selection for white tail spots in the barn swallow in relation to habitat choice by feather lice. Anim Behav 58:1201-1205

Møller AP (1994) Sexual selection and the barn swallow. Oxford University Press, Oxford

Møller AP (1995) Sexual selection in the barn swallow (Hirundo rustica). V. geographic variation in ornament size. J Evol Biol 8:3-19

Møller AP (2004) Rapid temporal change in frequency of infanticide in a passerine bird associated with change in population density and body condition. Behav Ecol 15:462-468

Møller AP, De Lope F, Saino N (1995) Sexual selection in the barn swallow Hirundo rustica.VI. Aerodynamic adaptations. J Evol Biol 8:671-687

Møller AP, Chabi Y, Cuervo JJ, De Lope F, Kilpimaa J, Kose M, Matyjasiak P, Pap PL, Saino N, Sakraoui R, Schifferli L, von Hirschheydt J (2006) An analysis of continent-wide patterns of sexual selection in a passerine bird. Evolution 60:856-868

Neuman CR, Safran RJ, Lovette IJ (2007) Male tail streamer length does not predict apparent or genetic reproductive success in North American barn swallows Hirundo rustica erythrogaster. J Avian Biol 38:28-36

R Development Core Team (2008) R: A language and environment for statistical computing. R Foundation for Statistical Computing, Vienna, Austria. ISBN 3-900051-07-0, URL http://www.Rproject.org

Safran RJ, McGraw KJ (2004) Plumage coloration, not length or symmetry of tail-streamers, is a sexually selected trait in North American barn swallows. Behav Ecol 15:455-461

Safran RJ, Adelman JS, McGraw KJ, Hau M (2008) Sexual signal exaggeration affects physiological state in male barn swallows. Curr Biol 18:R461-R462

Stearns SC (1992) The evolution of life histories. Oxford University Press, Oxford

Tanioka T (2007) Concise dictionary of Japanese place names, 5th edn. Sanseido, Tokyo (In Japanese)

Turner AK (2006) The barn swallow. Poyser, London

Vortman Y, Lottem A, Dor R, Lovette IJ, Safran RJ (2011) The sexual signals of the East-Mediterranean barn swallow: a different swallow tale. Behav Ecol 22:1344-1352

Wagner WE Jr, Beckers OM, Tolle AE, Basolo AL (2012) Tradeoffs limit the evolution of male traits that are attractive to females. Proc R Soc Lond B 279:2899-2906 